\title{
Health-Related Quality of Life and Health Utilities of Mild, Moderate, and Severe Asthma: Evidence from the Medical Expenditure Panel Survey
}

\author{
Hyun Jin Song $\mathbb{1}^{1,2}$ \\ Kathryn $\vee$ Blake $\mathbb{D D}^{3}$ \\ Debbie L Wilson (D) ${ }^{1,2}$ \\ Almut G Winterstein $\mathbb{D}^{1,2}$ \\ Haesuk Park (D) ${ }^{1,2}$ \\ 'Department of Pharmaceutical \\ Outcomes and Policy, College of \\ Pharmacy, University of Florida, \\ Gainesville, FL, USA; ${ }^{2}$ Center for Drug \\ Evaluation and Safety, University of \\ Florida, Gainesville, FL, USA; ${ }^{3}$ Center for \\ Pharmacogenomics and Translational \\ Research, Nemours Children's Health, \\ Jacksonville, FL, USA
}

Background: Little information is known about the health-related quality of life (HRQOL) and the patient's preference values by the severity of asthma. We evaluated the HRQOL and health utility impairment associated with asthma severity using the SF-12 and SF-6D.

Methods: We conducted a cross-sectional analysis of 2010-2016 Medical Expenditure Panel Survey database of asthma patients aged $\geq 18$ years and categorized them into mild, moderate, and severe asthma. Study outcomes included the SF-12 physical component summary (PCS) and mental component summary (MCS) for measuring HRQOL and SF$6 \mathrm{D}$ for health utility. Survey regression models were used to estimate HRQOL and utilities for mild, moderate, and severe asthma.

Results: Of 10,222 patients with asthma, $75.4 \%, 23.9 \%$, and $0.8 \%$ had mild, moderate and severe asthma. We observed that the greater the severity, the lower the SF-6D scores: 0.731 in mild, 0.723 in moderate, and 0.659 in severe asthma $(P<0.001)$. Patients with severe asthma had a significantly lower PCS compared to those with mild asthma $(-5.3 ; P<0.001)$ but there was no significant difference in MCS $(-1.9 ; P=0.309)$ controlling for socioeconomic and clinical variables. Asthma severity, women, older age, and having a lower level education and public insurance were significantly associated with lower PCS $(P<$ $0.01)$.

Conclusion: Asthma patients had worse physical HRQOL than mental health, especially patients with severe asthma. These data suggest that the management of physical health of female, older aged, and low education patients with asthma should be focused on improving HRQOL.

Keywords: asthma, health-related quality of life, mental health, physical limitation, severity, SF-6D, SF-12

\section{Introduction}

Asthma is the most common inflammatory disease of the lungs and is a complex disorder of the airways that causes the airways to narrow, leading to wheezing, shortness of breath, chest tightness, and coughing. ${ }^{1}$ In the United States (US), approximately 15.4 million people are treated for asthma each year, with a total annual cost of $\$ 81.9$ billion, including medical care, absenteeism, and mortality. ${ }^{2}$ The impact of asthma extends beyond these outcomes to include effects on life style, well-being, and perceived health status. As a result, asthma has been shown to impair a person's quality of life (QOL). ${ }^{3}$ The association between asthma and worsening health-related quality of life (HRQOL) in the general population is
Correspondence: Haesuk Park Department of Pharmaceutical Outcomes and Policy, College of Pharmacy,

University of Florida, 1225 Center Drive, Gainesville, FL, 3260I, USA

Tel + I 3522736261

Fax +I 3522736270

Email hpark@cop.ufl.edu 
well described. ${ }^{4-6}$ In the US, two recent studies using 2000-2002 Medical Expenditure Panel Survey (MEPS) data reported a 0.021 decreased utility score for patients with asthma compared to those without asthma using the EQ-5D scores of patients with asthma. ${ }^{7,8}$ A UK study estimated the HRQOL and health utilities of asthma patients using generic HRQOL questionnaires. ${ }^{9}$ Compared to patients without asthma, scores of the SF6D, EQ-5D, and visual analogue scale of those with asthma are decreased by $0.045,0.032$, and 4.64, respectively.

However, little is known about patient's HRQOL or health preference values (ie, utility weights) by severity level of asthma (ie, mild, moderate, and severe asthma) although it is known that people with severe asthma are usually refractory to standard treatment, and have poor symptom control affecting their social and work life. ${ }^{10}$ Previous clinical trials and observational studies using disease-specific tools in limited select populations (eg, uncontrolled severe persistent allergic asthma and refractory eosinophilic asthma) have reported that severe asthma impairs HRQOL. ${ }^{11}$ Although the narrow focus of asthma-specific instruments has the advantage of being potentially more sensitive to important changes in health due to health-care interventions, generic HRQOL and health utility measures can add additional benefits as they both are suitable for comparisons across disease states and the general population. ${ }^{12}$ To assess the impact of asthma on HRQOL at the population level, it is important to use comprehensive and valid measures of HRQOL suitable for implementation in the general population.

The 12-item short Form Health Survey (SF-12) Questionnaire, a generic HRQOL measure, has been found to be sensitive to clinically important differences between asthma patients who are not well controlled and those who are at least well controlled, where such control was determined using the validated Asthma Control Test whose levels align with the Global Initiative for Asthma (GINA) defined levels of asthma control. ${ }^{12-14}$ In addition, the SF-6D (ie, health utility weights) from the SF-12 enhances the evidence of the association between asthma and HRQOL by translating health outcomes into a preference-based measure (ie, value) for costeffectiveness studies. Thus, we estimated the HRQOL and health utilities of patients with asthma by the severity of asthma using SF-12 and SF-6D and identified factors associated with low HRQOL and health utilities in the general US population using nationally representative data.

\section{Methods}

\section{Data Source}

We used 2010-2016 MEPS data from the Agency for Healthcare Research and Quality and the National Center for Health Statistics. ${ }^{15}$ The MEPS Household Component is a nationally representative sample of the civilian noninstitutionalized population of the US for each calendar year. ${ }^{15}$ It includes person-level information on demographic characteristics, health-care use, health conditions, and HRQOL for each year of the National Health Interview Survey and provides nationally representative estimates for the US population using population weights. In the MEPS panel design, which uses a panel of respondents annually, a subsample of respondents is extracted from the previous year's National Health Interview Survey. The survey panels overlap and collect information from five rounds of interviews over a 2-year follow-up period to estimate health-care utilization and expenditures for each calendar year. ${ }^{15}$

\section{Definition of Asthma by Severity}

All persons aged $\geq 18$ years in each year from 2010 to 2016 were included in our analysis and were divided into patients with asthma and those without asthma. To identify asthma patients, we used the International Classification of Diseases, Ninth and Tenth Revision, Clinical Modification (ICD-9-CM: 493 and ICD-10-CM: J45) from the medical condition files for each year from 2010 to 2016. The control group (ie, patients without asthma) was defined as persons who did not have asthma diagnosis codes in each calendar year (ie, persons in all cohorts of each year excluding patients with asthma).

We classified patients with asthma into three groups by severity (mild, moderate, and severe asthma) according to the five management steps (steps 1 to 5) of the 2010-2016 GINA guidelines and previous studies. ${ }^{16-19}$ GINA guidelines categorize mild, moderate, and severe asthma using the five management steps when the patient has been on controller treatment for several months: steps 1 and 2 as mild, step 3 as moderate, and steps 4 and 5 as severe asthma. ${ }^{16}$ However, we had to categorize step 4 as moderate asthma, because there was limited information about the dose of inhaled corticosteroid/long-acting beta-agonist combinations (ICS/LABA) in MEPS. Thus, steps 3 and 4 
defined as moderate asthma to include low- to high-dose inhaled corticosteroid (ICS) (ie, low-dose ICS for step 3 and medium/high dose ICS for Step 4). ${ }^{19}$ In our study, mild, moderate, and severe asthma were defined using the medication prescription data as prescribed ICS or no prescribed asthma controller medication (steps 1 and 2 of GINA), prescribed ICS/LABA (steps 3 and 4 of GINA), and prescribed ICS/LABA and add-on omalizumab, mepolizumab, tiotropium, or low dose oral corticosteroid (OCS) (step 5 of GINA), respectively, as has been described in previously published studies. ${ }^{17-19}$ We defined low dose OCS for severe asthma based on a $\leq 7.5 \mathrm{mg}$ daily equivalent prednisolone for $\geq 2$ weeks using the strength of the OCS and days supplied or quantity of prescribed OCS. ${ }^{17-19}$

\section{Health-Related Quality of Life Measures}

We used self-reported outcomes including the SF-12 version 2 (SF-12v2) health survey, perceived overall and mental health, and physical limitation using the full year consolidated data file to estimate HRQOL of patients with asthma. To estimate HRQOL, we used the physical component summary (PCS) and mental component summary (MCS) scores of the SF-12v2 as reported in the MEPS data. The SF-12v2, a validated and widely used generic measure of HRQOL, is a questionnaire consisting of twelve questions that measure eight health domains to produce two summary scores representing physical and mental components of health. Physical health-related domains include general health, physical functioning, role physical, and body pain. Mental health-related domains include vitality, social functioning, role emotional, and mental health. ${ }^{20-22}$ PCS and MCS are constructed to have means of 50 and standard deviations of 10 using population norms, where a low score indicates low level of health status. ${ }^{23}$ In addition to the SF-12v2, overall and mental health were assessed using single questions (ie, "In general, would you say your health is" and "In general, would you say your mental health is") using five response anchors: excellent, very good, good, fair, and poor. Physical limitation was measured using a yes/no response anchor to a question regarding having difficulties walking, climbing stairs, grasping objects, reaching overhead, lifting, bending or stooping, or standing for long periods of time. We also calculated SF-6D scores using the SF-12v2 PCS and MCS score in the 2010-2016 MEPS data according to Hanmer's equation. ${ }^{24}$ The SF-6D is a health utility score with a metric anchored by 1 (perfect health) and 0 (death), where higher SF-6D scores represent better health. $^{21}$

\section{Statistical Analysis}

Descriptive statistics were used to present baseline characteristics of patients with and without asthma and we compared demographic and clinical variables of mild, moderate, and severe asthma groups with Rao-Scott chi-square tests and analysis of variance (ANOVA) for categorical and continuous variables, respectively. Among persons who completed data on each HRQOL scale, we compared PCS and MCS scores of the SF-12, perceived overall health and mental health, physical limitation, and SF-6D of (1) mild, moderate, and severe asthma compared to patients without asthma (2) and moderate and severe asthma compared to patients with mild asthma. We also compared the percentage of patients between asthma severity groups who reported having low quality of life in their self-reported perceived health status using Rao-Scott chi-square tests, where responses of fair or poor for the questions of perceived overall and mental health and of yes to the question of having physical limitations were considered low quality of life and all other responses were not considered to be low quality of life. We adjusted for the complex sample design to ensure nationally representative estimates. To obtain accurate estimates from the survey data, we used a survey regression model (SAS proc surveyreg), which incorporated person-level weights and variance adjustment weights (strata and primary sampling unit). ${ }^{15}$ The covariates used for adjustment in the regression models included the following: demographics (age, sex, race, Hispanic, region, marital status, education level, household income, and insurance) and clinical variables (smoking, Charlson Comorbidity Index [CCI], emergency department [ED] visits, and hospitalizations), which have been found to be associated with HRQOL. 5,25,26 We used the full year consolidated data, medical condition, emergency room visits, and hospital inpatient stay files to ascertain the covariates. To calculate the CCI score, we used ICD-9-CM and ICD-10-CM codes. $^{27,28}$ Statistical significances were defined as a 2 -sided $P<0.05$. All statistical analyses were performed using SAS version 9.4 (SAS Institute Inc., Cary, NC).

\section{Results}

\section{Descriptive Characteristics}

We identified 145,619 individuals, representing a population of about 1.5 billion non-institutionalized US 
civilians during 2010-2016, of which 7\% had asthma (Table 1). Of 10,222 patients with asthma, $75.4 \%$, $23.9 \%$, and $0.8 \%$ had mild, moderate, and severe asthma, respectively. The mean ages (standard errors [SE]) were 48.0 (0.4) in mild, 54.5 (0.6) in moderate, and 59.4 (2.0) in severe asthma. Patients with severe asthma were predominantly female $(78.8 \%)$, White $(68.8 \%)$, living in the south region $(50.0 \%)$, married $(45.0 \%)$, having a Bachelor's degree or more $(46.3 \%)$, and having private insurance $(66.3 \%)$, while those with mild asthma were more likely to have been smokers (19.1\%). In addition, those with severe asthma were more likely to have visited the ED $(33.8 \%)$ or to have been hospitalized (18.8\%) during the 2010-2016 period. The CCI scores (SE) increased as the severity of asthma increased: $0.70(0.03)$ in mild, 0.85 $(0.04)$ in moderate, and $1.29(0.31)$ in severe asthma.

\section{Perceived Health Status}

Figure 1 shows self-reported overall health, mental health, and physical limitation by presence of asthma and severity of asthma. The percentage of patients reporting low quality of life (fair or poor) in overall health increased as the severity of asthma increased; $27.6 \%, 32.3 \%$, and $37.1 \%$ of mild, moderate, and severe asthma, respectively $(P<$ 0.001). A similar trend was observed in the percentage of patients reporting physical limitations with $66.1 \%$ of patients with severe asthma having physical limitation compared to $26.7 \%$ of patients with mild asthma $(P<$ $0.001)$. The percentage of patients with severe asthma reporting low quality of life in mental health was not statistically significant compared to mild asthma $(18.2 \%$ vs $16.7 \%, P=0.640)$.

\section{SF-12 PCS and MCS}

Table 2 presents the SF-12 PCS and MCS scores of physical and mental health from the twelve questions of the SF-12 of patients with and without asthma, categorized by asthma severity and demographic characteristics. Overall, as compared to patients without asthma, those with asthma had a significant reduction of HRQOL, with a mean of -5.3 for PCS (43.2 vs 48.5) and -2.6 for MCS (48.4 vs 51.0) $(P<0.001)$. Among patients with asthma, the mean PCS score was lower than that of the MCS score (43.2 vs 48.4) $(P<0.001)$. The mean PCS was significantly lower in severe asthma (35.9) patients, compared to those with mild (43.9) and moderate asthma (41.6). We observed a similar trend for the mean MCS score (48.2 in mild, 49.2 in moderate, and 47.1 in severe asthma $)(P<0.001)$ although the group differences were smaller than those for the PCS score. In subgroup analyses, the association between severe asthma and lower PCS and MCS scores remained consistent among men and women, all races, and across age groups except for the age group of 18-34 years (Table 2). Table 3 presents results from the multivariable survey regression of SF-12 PCS and MCS controlling for demographics and clinical characteristics in patients with moderate and severe asthma compared to mild asthma. After controlling for covariates, the PCS scores of moderate and severe asthma were significantly decreased compared to mild asthma $(P<0.05)$, but not for the MCS scores in moderate $(P=0.252)$ and severe asthma $(P=$ 0.309). The risk factors associated with low PCS and MCS scores were ED visits, hospitalizations, lower income level, smoker, and higher CCI score $(P<0.01)$. In particular, older age, female, region, low education level, and public insurance were significantly associated with lower scores of PCS $(P<0.01)$, but not of MCS.

\section{SF-6D}

The mean SF-6D scores of patients with and without asthma were 0.728 and 0.794 . Among patients with asthma, we found that the greater the severity, the lower the SF-6D scores: 0.731 in mild, 0.723 in moderate, and 0.659 in severe asthma $(P<0.001)$ (Table 4). Examining subgroups of men and women, age groups of $\geq 35$ years, and races of white and black, we noted a trend of significantly decreasing utility values as the severity of asthma increased $(P<0.001)$. Table 5 shows increased utility weights or disutilities of the SF-6D among patients with asthma by demographic and clinical factors after controlling for covariates. Compared to mild asthma, the greater the increase in the asthma severity level, the higher the disutility; -0.003 in moderate and -0.059 in severe asthma $(P=0.001)$, although the disutility of moderate asthma was not statistically significant $(P=0.482)$. Older age, female, living in the Midwest, South, and West, lower education level, lower income level, public insurance, smoking, and higher CCI were factors significantly associated with lower SF-6D scores $(P<0.01)$. ED visits $(-0.039)$ and hospitalizations $(-0.042)$ had an adverse association on utilities $(P<0.001)$.

\section{Discussion}

To our best knowledge, this is the first study to measure the HRQOL and health utilities by severity of asthma using a nationally representative US sample. Our results 
Table I Baseline Characteristics of Patients without Asthma and Patients with Asthma by Severity, 2010-2016 ${ }^{\mathrm{a}}$

\begin{tabular}{|c|c|c|c|c|c|c|}
\hline & $\begin{array}{l}\text { No Asthma } \\
(n=135,397)\end{array}$ & $\begin{array}{l}\text { Total Asthma } \\
(n=10,222)\end{array}$ & $\begin{array}{l}\text { Mild Asthma } \\
(\mathrm{n}=7704)\end{array}$ & $\begin{array}{l}\text { Moderate Asthma } \\
(n=2438)\end{array}$ & $\begin{array}{l}\text { Severe Asthma } \\
(\mathbf{n}=\mathbf{8 0})\end{array}$ & $P_{\text {-value }}$ \\
\hline National estimates, $\mathrm{N}(\%)$ & $1,418,534,135$ & $105,740,815$ & $78,021,972$ & $26,630,773$ & $1,088,070$ & \\
\hline Age (years), mean (SE) & $49.1(0.2)$ & $49.7(0.4)$ & $48.0(0.4)$ & $54.5(0.6)$ & $59.4(2.0)$ & $<0.001$ \\
\hline $18-34$ & $36,566(27.0 \%)$ & $2492(24.4 \%)$ & $2150(27.9 \%)$ & 339 (13.9\%) & $3(3.8 \%)$ & $<0.001$ \\
\hline $35-64$ & 71,068 (52.5\%) & $5496(53.8 \%)$ & $4060(52.7 \%)$ & I $388(56.9 \%)$ & $48(60.0 \%)$ & \\
\hline$\geq 65$ & $27,763(20.5 \%)$ & $2234(21.9 \%)$ & 1494 (19.4\%) & 711 (29.2\%) & $29(36.3 \%)$ & \\
\hline Sex & & & & & & $<0.001$ \\
\hline Male & $58,750(43.4 \%)$ & $3300(32.3 \%)$ & $2466(32.0 \%)$ & 817 (33.5\%) & 17 (21.3\%) & \\
\hline Female & 76,647 (56.6\%) & $6922(67.7 \%)$ & $5238(68.0 \%)$ & 1621 (66.5\%) & $63(78.8 \%)$ & \\
\hline Race & & & & & & $<0.001$ \\
\hline White & 94,434 (69.7\%) & 6702 (65.6\%) & 4976 (64.6\%) & 1671 (68.5\%) & $55(68.8 \%)$ & \\
\hline Black & $26,743(19.8 \%)$ & $2527(24.7 \%)$ & $1970(25.6 \%)$ & $538(22.1 \%)$ & $19(23.8 \%)$ & \\
\hline Other $^{c}$ & $1133(0.8 \%)$ & $120(1.2 \%)$ & $92(1.2 \%)$ & $28(1.1 \%)$ & $0(0 \%)$ & \\
\hline Hispanic & & & & & & $<0.001$ \\
\hline Yes & $32,496(24.0 \%)$ & 2002 (19.6\%) & $1624(21.1 \%)$ & 375 (15.4\%) & $3(3.8 \%)$ & \\
\hline No & $102,90 \mid(76.0 \%)$ & $8220(80.4 \%)$ & $6080(78.9 \%)$ & $2063(84.6 \%)$ & $77(96.3 \%)$ & \\
\hline Region & & & & & & $<0.001$ \\
\hline Northwest & $22,098(16.3 \%)$ & $2144(21.0 \%)$ & $1588(20.6 \%)$ & $540(22.1 \%)$ & $16(20.0 \%)$ & \\
\hline Midwest & $27,60 \mathrm{I}(20.4 \%)$ & $2183(21.4 \%)$ & $1617(21.0 \%)$ & 550 (22.6\%) & $16(20.0 \%)$ & \\
\hline South & 49,931 (36.9\%) & $3469(33.9 \%)$ & $2571(33.4 \%)$ & 858 (35.2\%) & $40(50.0 \%)$ & \\
\hline West & $35,767(26.4 \%)$ & $2426(23.7 \%)$ & $1928(25.0 \%)$ & $490(20.1 \%)$ & $8(10.0 \%)$ & \\
\hline Marital status & & & & & & $<0.001$ \\
\hline Married & $67,425(49.8 \%)$ & $4154(40.6 \%)$ & $3040(39.5 \%)$ & $1078(44.2 \%)$ & $36(45.0 \%)$ & \\
\hline Widowed & $10,019(7.4 \%)$ & $970(9.5 \%)$ & $671(8.7 \%)$ & $290(11.9 \%)$ & $9(11.3 \%)$ & \\
\hline Divorced/separated & $21,148(15.6 \%)$ & $2075(20.3 \%)$ & $1529(19.8 \%)$ & $527(21.6 \%)$ & $19(23.8 \%)$ & \\
\hline Not married & $36,804(27.2 \%)$ & $3023(29.6 \%)$ & $2464(32.0 \%)$ & $543(22.3 \%)$ & $16(20.0 \%)$ & \\
\hline Education & & & & & & $<0.001$ \\
\hline Less than high school diploma & $25,640(18.9 \%)$ & $2182(21.3 \%)$ & $1689(21.9 \%)$ & 484 (19.9\%) & $9(11.3 \%)$ & \\
\hline High school diploma & $36,133(26.7 \%)$ & $2647(25.9 \%)$ & $1973(25.6 \%)$ & $649(26.6 \%)$ & $25(31.3 \%)$ & \\
\hline Bachelor's degree & $50,525(37.3 \%)$ & $3765(36.8 \%)$ & $2808(36.4 \%)$ & $920(37.7 \%)$ & $37(46.3 \%)$ & \\
\hline Advanced degree & II,645 (8.6\%) & $837(8.2 \%)$ & $603(7.8 \%)$ & $227(9.3 \%)$ & $7(8.8 \%)$ & \\
\hline Unknown & $11,454(8.5 \%)$ & 791 (7.7\%) & $631(8.2 \%)$ & $158(6.5 \%)$ & $2(2.5 \%)$ & \\
\hline Income level & & & & & & $<0.001$ \\
\hline$\leq 0$ & $3487(2.6 \%)$ & 349 (3.4\%) & $280(3.6 \%)$ & $64(2.6 \%)$ & $5(6.3 \%)$ & \\
\hline$>0-20,000$ & $28,397(21.0 \%)$ & $2963(29.0 \%)$ & $2270(29.5 \%)$ & $673(27.6 \%)$ & $20(25.0 \%)$ & \\
\hline$>20,000-40,000$ & $29,915(22.1 \%)$ & $2230(21.8 \%)$ & $1692(22.0 \%)$ & $519(21.3 \%)$ & $19(23.8 \%)$ & \\
\hline$>40,000-80,000$ & $37,217(27.5 \%)$ & $2446(23.9 \%)$ & $1838(23.9 \%)$ & $592(24.3 \%)$ & $16(20.0 \%)$ & \\
\hline$>80,000$ & 36,381 (26.9\%) & $2234(21.9 \%)$ & $1624(21.1 \%)$ & $590(24.2 \%)$ & $20(25.0 \%)$ & \\
\hline Insurance & & & & & & $<0.001$ \\
\hline Any private & $80,708(59.6 \%)$ & $5378(52.6 \%)$ & $3958(51.4 \%)$ & $1367(56.1 \%)$ & $53(66.3 \%)$ & \\
\hline Public only & 36,337 (26.8\%) & $4000(39.1 \%)$ & $3017(39.2 \%)$ & 957 (39.3\%) & $26(32.5 \%)$ & \\
\hline Uninsured & $18,352(13.6 \%)$ & $844(8.3 \%)$ & 729 (9.5\%) & 114 (4.7\%) & I (1.3\%) & \\
\hline Smoking status & & & & & & $<0.001$ \\
\hline Yes & 20,818 (15.4\%) & $1893(18.5 \%)$ & 1474 (19.1\%) & 409 (16.8\%) & $10(12.5 \%)$ & \\
\hline No & $101,529(75.0 \%)$ & 7389 (72.3\%) & $5484(71.2 \%)$ & 1839 (75.4\%) & $66(82.5 \%)$ & \\
\hline Unknown & $13,050(9.6 \%)$ & 940 (9.2\%) & 746 (9.7\%) & $190(7.8 \%)$ & $4(5.0 \%)$ & \\
\hline
\end{tabular}

(Continued) 
Table I (Continued).

\begin{tabular}{|l|l|l|l|l|l|l|}
\hline & $\begin{array}{l}\text { No Asthma } \\
(\mathbf{n}=135,397)\end{array}$ & $\begin{array}{l}\text { Total Asthma } \\
(\mathbf{n}=1 \mathbf{1 0 2 2 2})\end{array}$ & $\begin{array}{l}\text { Mild Asthma } \\
(\mathbf{n}=\mathbf{7 7 0 4})\end{array}$ & $\begin{array}{l}\text { Moderate Asthma } \\
(\mathbf{n}=\mathbf{2 4 3 8})\end{array}$ & $\begin{array}{l}\text { Severe Asthma } \\
(\mathbf{n}=\mathbf{8 0})\end{array}$ & $\begin{array}{l}\text { P-value } \\
\text { b }\end{array}$ \\
\hline ED visits & $24,332(18.0 \%)$ & $2993(29.3 \%)$ & $2252(29.2 \%)$ & $714(29.3 \%)$ & $27(33.8 \%)$ & $<0.001$ \\
\hline Hospitalizations & $13,249(9.8 \%)$ & $1547(15.1 \%)$ & $1074(13.9 \%)$ & $458(18.8 \%)$ & $15(18.8 \%)$ & $<0.001$ \\
\hline $\mathrm{CCl}$, mean (SE) & $0.51(0.01)$ & $0.74(0.02)$ & $0.70(0.03)$ & $0.85(0.04)$ & $1.29(0.31)$ & $<0.001$ \\
\hline
\end{tabular}

Notes: ${ }^{a}$ Data were presented as the number of patients (percentage). ${ }^{b}$ Statistical significance was compared between patients with mild, moderate, severe asthma with a 2-sided $P<0.05$ using Rao-Scott Chi-square test and ANOVA. 'Other includes American Indian, Alaska Native, Asian, Native Hawaiian, Pacific Islander, and multiple races. ${ }^{d}$ Charlson Comorbidity Index includes myocardial infarction, heart failure, peripheral vascular disease, dementia, cerebrovascular disease, connective tissue disease, ulcer disease mild liver disease hemiplegia, moderate or severe renal disease, diabetes, tumor, moderate or severe liver, and metastatic solid tumor.

Abbreviations: $\mathrm{SE}$, standard error; $\mathrm{CCl}$, charlson comorbidity index.

among 105 million patients with asthma indicate that the HRQOL of the physical health is significantly worsened in patients with severe asthma using a validated general HRQOL tool (ie, SF-12 PCS and MCS) as well as using self-perceived health status. In the self-reported perceived health status, the patients with asthma were 2 times more likely to perceive that their overall health was fair or poor compared to those without asthma. Indeed, more than a third of the patients with severe asthma rated their overall health as impaired.
The SF-12 has been shown to be a valid evaluation tool with excellent measurement in patients with asthma. ${ }^{12}$ Lower scores for the PCS than the MCS in patients with asthma have also been reported by other studies. ${ }^{22,29}$ Our results confirm that asthma is associated with not only declining HRQOL using the validated HRQOL tool (ie, SF-12 PCS and MCS), but also a higher physical health deterioration than mental health. Further, we found that patients with moderate and severe asthma had significantly lower PCS compared to those

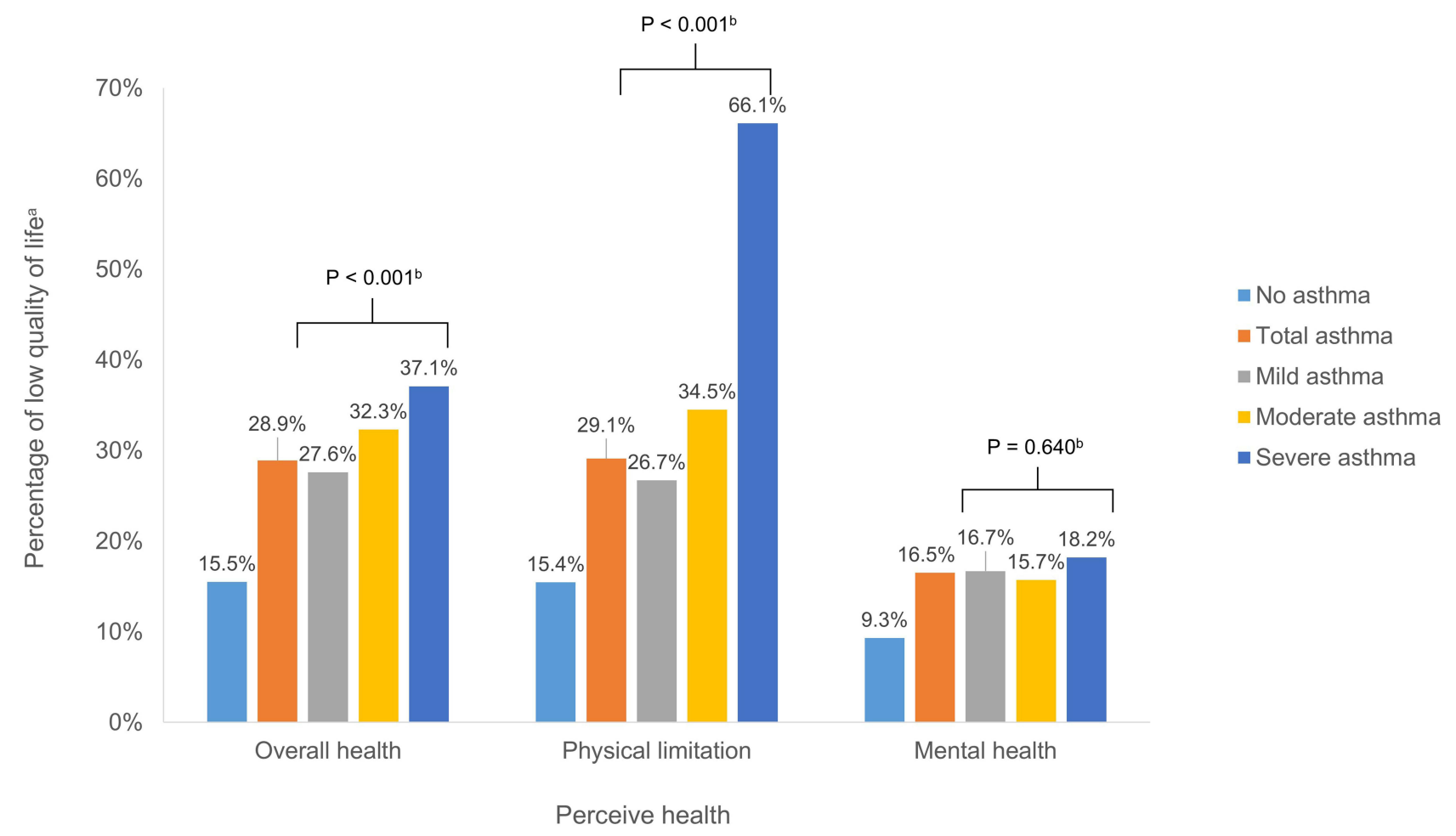

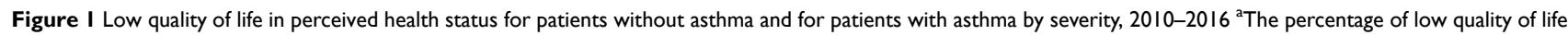
defined as the percentage of persons who answered fair or poor for the question of perceived overall and mental health and the percentage of persons who reported physical limitation by severity of asthma. ${ }^{b}$ Statistical significance was compared between patients with mild, moderate, severe asthma with a 2 -sided $P<0.05$ using Rao-Scott chi-square test. 
Table 2 SF-I 2 Physical and Mental Health Summary Scores of Patients without Asthma and Patients with Asthma by Severity, 20102016

\begin{tabular}{|c|c|c|c|c|c|c|c|c|c|c|c|}
\hline & \multicolumn{2}{|c|}{$\begin{array}{l}\text { No Asthma } \\
(n=123,455)\end{array}$} & \multicolumn{2}{|c|}{$\begin{array}{l}\text { Total Asthma } \\
(n=9388)\end{array}$} & \multicolumn{2}{|c|}{$\begin{array}{l}\text { Mild } \\
(n=7042)\end{array}$} & \multicolumn{2}{|c|}{$\begin{array}{l}\text { Moderate } \\
(n=2274)\end{array}$} & \multicolumn{2}{|c|}{$\begin{array}{l}\text { Severe } \\
(n=77)\end{array}$} & \multirow[t]{2}{*}{ P-value ${ }^{a}$} \\
\hline & Mean & (SE) & Mean & (SE) & Mean & (SE) & Mean & (SE) & Mean & (SE) & \\
\hline \multicolumn{12}{|l|}{ SF-12, PCS } \\
\hline Total & 48.5 & $(0.1)$ & 43.2 & $(0.3)$ & 43.9 & $(0.3)$ & 41.6 & $(0.5)$ & 35.9 & $(1.3)$ & $<0.001$ \\
\hline \multicolumn{12}{|l|}{ Sex } \\
\hline Men & 49.0 & $(0.1)$ & 44.8 & $(0.4)$ & 45.4 & $(0.4)$ & 43.5 & $(0.7)$ & 37.6 & (2.9) & $<0.001$ \\
\hline Women & 48.1 & $(0.1)$ & 42.4 & $(0.3)$ & 43.2 & $(0.3)$ & 40.5 & $(0.6)$ & 35.4 & (I.4) & $<0.001$ \\
\hline \multicolumn{12}{|l|}{ Age (years) } \\
\hline $18-34$ & 53.2 & $(0.1)$ & 50.2 & $(0.4)$ & 50.3 & $(0.4)$ & 49.4 & $(1.0)$ & 51.4 & $(0.6)$ & $<0.001$ \\
\hline $35-64$ & 48.7 & $(0.1)$ & 42.7 & $(0.3)$ & 42.9 & (0.4) & 42.5 & $(0.5)$ & 33.9 & (I.5) & $<0.001$ \\
\hline$\geq 65$ & 42.1 & $(0.2)$ & 36.6 & $(0.5)$ & 36.8 & $(0.5)$ & 36.2 & $(0.9)$ & 36.8 & (I.7) & $<0.001$ \\
\hline \multicolumn{12}{|l|}{ Race } \\
\hline White & 48.6 & $(0.1)$ & 43.3 & $(0.3)$ & 44.0 & $(0.3)$ & 41.7 & $(0.5)$ & 36.1 & (1.3) & $<0.001$ \\
\hline Black & 47.2 & $(0.2)$ & 42.6 & $(0.4)$ & 43.5 & $(0.4)$ & 39.7 & $(0.8)$ & 34.2 & $(4.0)$ & $<0.001$ \\
\hline Other ${ }^{b}$ & 49.5 & $(0.2)$ & 43.6 & $(1.0)$ & 44.1 & $(1.2)$ & 42.1 & $(1.2)$ & 35.6 & (3.3) & $<0.001$ \\
\hline \multicolumn{12}{|l|}{ Smoking } \\
\hline Yes & 46.5 & $(0.1)$ & 39.7 & $(0.6)$ & 40.6 & $(0.6)$ & 36.9 & $(1.0)$ & 34.7 & $(4.2)$ & $<0.001$ \\
\hline No & 48.9 & $(0.1)$ & 44.1 & $(0.3)$ & 44.8 & $(0.3)$ & 42.7 & $(0.5)$ & 36.0 & (1.4) & $<0.001$ \\
\hline \multicolumn{12}{|l|}{ SF-I2, MCS } \\
\hline Total & 51.0 & $(0.1)$ & 48.4 & $(0.2)$ & 48.2 & $(0.2)$ & 49.2 & $(0.3)$ & 47.1 & (2.0) & $<0.001$ \\
\hline \multicolumn{12}{|l|}{ Sex } \\
\hline Men & 51.8 & $(0.1)$ & 49.5 & $(0.3)$ & 49.2 & $(0.3)$ & 50.4 & $(0.6)$ & 46.0 & (3.3) & $<0.001$ \\
\hline Women & 50.3 & $(0.1)$ & 47.9 & $(0.2)$ & 47.7 & $(0.3)$ & 48.6 & $(0.4)$ & 47.4 & (2.4) & $<0.001$ \\
\hline \multicolumn{12}{|l|}{ Age (years) } \\
\hline $18-34$ & 49.9 & $(0.1)$ & 48.2 & $(0.4)$ & 48.3 & $(0.5)$ & 48.0 & (I.I) & 52.7 & $(0.8)$ & $<0.001$ \\
\hline $35-64$ & 50.5 & $(0.1)$ & 47.2 & $(0.3)$ & 47.0 & $(0.3)$ & 47.8 & $(0.5)$ & 47.0 & $(2.9)$ & $<0.001$ \\
\hline$\geq 65$ & 52.4 & $(0.1)$ & 50.7 & $(0.4)$ & 50.2 & $(0.5)$ & 51.8 & $(0.5)$ & 46.6 & $(3.1)$ & $<0.001$ \\
\hline \multicolumn{12}{|l|}{ Race } \\
\hline White & 51.0 & $(0.1)$ & 50.8 & $(0.5)$ & 48.2 & $(0.3)$ & 49.4 & $(0.4)$ & 47.0 & (2.4) & $<0.001$ \\
\hline Black & 50.9 & $(0.1)$ & 48.3 & $(0.3)$ & 48.7 & $(0.3)$ & 47.1 & $(0.9)$ & 46.4 & (3.6) & $<0.001$ \\
\hline Other $^{b}$ & 51.7 & $(0.2)$ & 48.2 & $(0.6)$ & 47.8 & $(0.6)$ & 49.5 & (I.3) & 48.2 & $(0.6)$ & $<0.001$ \\
\hline \multicolumn{12}{|l|}{ Smoking } \\
\hline Yes & 47.6 & $(0.1)$ & 44.3 & $(0.5)$ & 44.3 & $(0.5)$ & 44.3 & (1.0) & 48.8 & $(4.2)$ & $<0.001$ \\
\hline No & 51.6 & $(0.1)$ & 49.5 & $(0.2)$ & 49.2 & $(0.2)$ & 50.3 & $(0.3)$ & 46.9 & $(2.2)$ & $<0.001$ \\
\hline
\end{tabular}

Notes: ${ }^{a}$ Statistical significance was compared between patients with mild, moderate, severe asthma with a 2 -sided $P<0.05$ using ANOVA. ${ }^{\mathrm{b}}$ Other includes American Indian, Alaska Native, Asian, Native Hawaiian, Pacific Islander, and multiple races.

Abbreviations: SF-12v2, I2-Item short form health survey version 2; PCS, physical component summary; MCS, mental component summary; SE, standard error.

with mild asthma but there were no significant differences in MCS by the severity of asthma after controlling for covariates. Poor lung function of patients with asthma can lead to poorer physical functioning compared to those without asthma. ${ }^{30}$ In other work, the HRQOL impairment of SF-12 PCS in adults with respiratory symptoms including wheezing, chest tightness, breathlessness, and cough were higher than the impairment of their MCS. ${ }^{31}$ In particular, severe asthma, described as having intense symptoms and airflow limitation, has been associated with a HRQOL burden due to excessive symptoms, frequent and life-threatening attacks, increased 
Table 3 Multivariable Survey Regression Analysis to Estimate the SF-I2 PCS and MCS of Asthma Patients by Severity, 20I0-20I6

\begin{tabular}{|c|c|c|c|c|c|c|}
\hline & \multicolumn{3}{|c|}{ SF-I 2 PCS } & \multicolumn{3}{|c|}{ SF-I 2 MCS } \\
\hline & Coefficient & SE & P-value & Coefficient & SE & P-value \\
\hline \multicolumn{7}{|l|}{ Asthma severity (ref: mild asthma) } \\
\hline Moderate & -0.966 & 0.381 & 0.012 & 0.459 & 0.399 & 0.252 \\
\hline Severe & -5.273 & 1.344 & $<0.001$ & -1.875 & 1.837 & 0.309 \\
\hline ED visits & -2.637 & 1.344 & $<0.001$ & -1.987 & 0.406 & $<0.001$ \\
\hline Hospitalizations & -3.605 & 0.566 & $<0.001$ & -1.518 & 0.491 & 0.002 \\
\hline \multicolumn{7}{|l|}{ Age (years) (ref: 18-34) } \\
\hline $35-64$ & -6.049 & 0.398 & $<0.001$ & -0.940 & 0.454 & 0.040 \\
\hline$\geq 65$ & -8.446 & 0.630 & $<0.001$ & 4.041 & 0.601 & $<0.001$ \\
\hline \multicolumn{7}{|l|}{ Sex (ref: male) } \\
\hline Female & -1.076 & 0.359 & 0.003 & -0.883 & 0.374 & 0.019 \\
\hline \multicolumn{7}{|l|}{ Race (ref: White) } \\
\hline Black & 1.364 & 0.371 & $<0.001$ & 2.342 & 0.399 & $<0.001$ \\
\hline Other ${ }^{\mathrm{a}}$ & -0.350 & $0.74 I$ & 0.638 & -0.287 & 0.545 & 0.600 \\
\hline \multicolumn{7}{|l|}{ Hispanic (ref: no) } \\
\hline Yes & 1.385 & 0.447 & 0.002 & 0.724 & 0.443 & 0.104 \\
\hline \multicolumn{7}{|l|}{ Region (ref: northwest) } \\
\hline Midwest & -2.164 & 0.552 & $<0.001$ & -0.302 & 0.514 & 0.558 \\
\hline South & -2.046 & 0.526 & $<0.001$ & -0.634 & 0.480 & 0.188 \\
\hline West & -1.733 & 0.569 & 0.003 & -0.730 & 0.541 & 0.179 \\
\hline \multicolumn{7}{|l|}{ Marital status (ref: not married) } \\
\hline Married & -0.383 & 0.669 & 0.568 & -0.179 & 0.626 & 0.776 \\
\hline Widowed & 0.830 & 0.524 & 0.115 & -1.609 & 0.545 & 0.004 \\
\hline Divorced/separated & 1.873 & 0.485 & $<0.001$ & -0.395 & 0.511 & 0.440 \\
\hline \multicolumn{7}{|l|}{ Education (ref: advanced degree) } \\
\hline Less than high school diploma & -4.186 & 0.727 & $<0.001$ & -1.357 & 0.592 & 0.023 \\
\hline High school graduate & -3.540 & 0.667 & $<0.001$ & 0.238 & 0.619 & 0.701 \\
\hline Bachelor's degree & -2.470 & 0.531 & $<0.001$ & -0.165 & 0.567 & 0.771 \\
\hline \multicolumn{7}{|l|}{ Income level (ref: $>80,000)$} \\
\hline$\leq 20,000$ & -5.011 & 0.532 & $<0.001$ & -3.893 & 0.565 & $<0.001$ \\
\hline$>20,000-40,000$ & -3.303 & 0.496 & $<0.001$ & -2.612 & 0.529 & $<0.001$ \\
\hline$>40,000-80,000$ & -2.098 & 0.426 & $<0.001$ & -0.740 & 0.440 & 0.094 \\
\hline \multicolumn{7}{|l|}{ Insurance (ref: uninsured) } \\
\hline Any private & 1.168 & 0.697 & 0.095 & 2.937 & 0.828 & 0.001 \\
\hline Public only & -3.817 & 0.755 & $<0.001$ & -0.863 & 0.887 & 0.332 \\
\hline Smoking & -1.512 & 0.498 & 0.003 & -2.037 & 0.514 & $<0.001$ \\
\hline $\mathrm{CCl}^{\mathrm{b}}$ & -1.831 & 0.150 & $<0.001$ & -0.689 & 0.138 & $<0.001$ \\
\hline Intercept & 58.605 & 1.020 & $<0.001$ & 50.978 & $\mathrm{I} .097$ & $<0.001$ \\
\hline
\end{tabular}

Notes: ${ }^{a}$ Other includes American Indian, Alaska Native, Asian, Native Hawaiian, Pacific Islander, and multiple races. ${ }^{\mathrm{b}}$ Charlson Comorbidity Index includes myocardial infarction, heart failure, peripheral vascular disease, dementia, cerebrovascular disease, connective tissue disease, ulcer disease mild liver disease hemiplegia, moderate or severe renal disease, diabetes, tumor, moderate or severe liver, and metastatic solid tumor.

Abbreviations: SF-6D, short-form six-dimension; PCS, physical component summary; MCS, mental component summary; SE, standard error; ED, emergency department; $\mathrm{CCl}$, charlson comorbidity index.

comorbidity burden, and high pharmacological treatment requirement. ${ }^{1,19,32,33}$ Moreover, previous studies reported that the two main domains that potentially affect the
HRQOL in patients with severe asthma are symptom and activity limitations, which result in worse physical HRQOL components than those of mental health. ${ }^{11,34}$ 
Table 4 SF-6D Scores of Patients without Asthma and Patients with Asthma by Severity, 2010-2016

\begin{tabular}{|c|c|c|c|c|c|c|c|c|c|c|c|}
\hline & \multicolumn{2}{|c|}{$\begin{array}{l}\text { No Asthma } \\
(n=|23,39|)\end{array}$} & \multicolumn{2}{|c|}{$\begin{array}{l}\text { Total Asthma } \\
(n=9387)\end{array}$} & \multicolumn{2}{|c|}{$\begin{array}{l}\text { Mild } \\
(n=7037)\end{array}$} & \multicolumn{2}{|c|}{$\begin{array}{l}\text { Moderate } \\
(n=2273)\end{array}$} & \multicolumn{2}{|c|}{$\begin{array}{l}\text { Severe } \\
(n=77)\end{array}$} & \multirow[t]{2}{*}{ P-value ${ }^{a}$} \\
\hline & Mean & (SE) & Mean & (SE) & Mean & (SE) & Mean & (SE) & Mean & (SE) & \\
\hline Total & 0.794 & $(0.001)$ & 0.728 & $(0.003)$ & 0.731 & $(0.003)$ & 0.723 & $(0.005)$ & 0.659 & $(0.023)$ & $<0.001$ \\
\hline \multicolumn{12}{|l|}{ Sex } \\
\hline Men & 0.807 & $(0.00 I)$ & 0.753 & $(0.005)$ & 0.755 & $(0.006)$ & 0.751 & $(0.009)$ & 0.665 & $(0.042)$ & $<0.001$ \\
\hline Women & 0.783 & $(0.00 I)$ & 0.716 & $(0.004)$ & 0.719 & $(0.004)$ & 0.708 & $(0.006)$ & 0.658 & $(0.027)$ & $<0.001$ \\
\hline \multicolumn{12}{|l|}{ Age (years) } \\
\hline $18-34$ & 0.816 & $(0.001)$ & 0.777 & $(0.005)$ & 0.778 & $(0.005)$ & 0.769 & $(0.014)$ & 0.830 & $(0.007)$ & $<0.001$ \\
\hline $35-64$ & 0.791 & $(0.00 I)$ & 0.713 & $(0.004)$ & 0.713 & $(0.005)$ & 0.716 & $(0.007)$ & $0.64 I$ & $(0.033)$ & $<0.001$ \\
\hline$\geq 65$ & 0.760 & $(0.002)$ & 0.701 & $(0.006)$ & 0.698 & $(0.007)$ & 0.708 & $(0.009)$ & 0.663 & $(0.030)$ & $<0.001$ \\
\hline \multicolumn{12}{|l|}{ Race } \\
\hline White & 0.794 & $(0.001)$ & 0.730 & $(0.004)$ & 0.732 & $(0.004)$ & 0.726 & $(0.006)$ & 0.660 & $(0.026)$ & $<0.001$ \\
\hline Black & 0.782 & $(0.002)$ & 0.722 & $(0.005)$ & 0.732 & $(0.005)$ & 0.688 & $(0.012)$ & 0.638 & $(0.054)$ & $<0.001$ \\
\hline Other & 0.808 & $(0.003)$ & 0.729 & $(0.011)$ & 0.729 & $(0.013)$ & 0.730 & $(0.016)$ & 0.668 & $(0.032)$ & $<0.001$ \\
\hline \multicolumn{12}{|l|}{ Smoking } \\
\hline Yes & 0.747 & $(0.002)$ & 0.661 & $(0.007)$ & 0.668 & $(0.007)$ & 0.640 & $(0.0 \mid 2)$ & 0.665 & $(0.034)$ & $<0.001$ \\
\hline No & 0.803 & $(0.00 I)$ & 0.745 & $(0.003)$ & 0.747 & $(0.003)$ & 0.743 & $(0.005)$ & 0.658 & $(0.027)$ & $<0.001$ \\
\hline
\end{tabular}

Notes: ${ }^{a}$ Statistical significance was compared between patients with mild, moderate, severe asthma with a 2 -sided $P<0.05$ using ANOVA. Abbreviations: SF-6D, short-form six-dimension; SE, standard error.

Consistent with previous studies, ${ }^{11,35}$ our study suggested that severe asthma significantly influences HRQOL. Although there was a study that showed significantly worse utilities using EQ-5D in patients who experienced an exacerbation compared to those who did not, their findings were limited because of the small sample size of patients who experienced an exacerbation with and without hospitalization $(n=22$ and $n=5) .{ }^{36}$ Our results using SF-6D suggest that the greater the severity of asthma, the worse the health utilities. Recent studies reported worse HRQOL in patients with severe asthma using a disease-specific HRQOL questionnaire. ${ }^{11}$ Among the asthma-specific measures, the Sydney Quality of Life Questionnaire (AQLQ-S), a nonutility-based asthma-specific QOL instrument consists of four domains: breathlessness, mood disturbance, social disruption, and concerns for health. ${ }^{36}$ In addition to the four comparable domains (ie, physical functioning, vitality, role functioning, social functioning, mental health) in the asthma-specific tool, the SF-6D also includes the pain domain, and thus consists of six dimensions, as the domain of the social disruption of the AQLQ-S is comparable to the two domains of role functioning and social functioning of the SF-6D. ${ }^{36}$ While AQLQ-S is the shortest asthma-specific questionnaire and is suitable for use in population-based studies with large numbers of patients, the Juniper AQLQ (AQLQ-J) is practical and convenient for using the questionnaire in multinational studies, and has been linguistically validated in several languages. ${ }^{37}$ The AQLQ-J was developed for use in clinical trials and includes a component in the activity domain that assesses individualized activity limitations by asking the respondent to choose the five activities that are the most important to them, ${ }^{38}$ thus the AQLQ-J is most likely better suited to measuring HRQOL in an asthma clinical trial than in a large population based study. In this respect, it is recommended that generic instruments continue to be used alongside diseasespecific instruments. ${ }^{12}$ Although generic measures may be slightly less responsive to changes in asthma symptoms than disease-specific measures, they can capture the general functional status associated with unexpected or additional side effects of asthma (eg, bodily pain). ${ }^{12,26,36}$ A limitation of the disease-specific instruments for economic evaluation is that the HRQOL from disease-specific measures cannot be converted to utility weights and therefore cannot be used to estimate quality-adjusted life years (QALYs), which includes both the quality and the quantity of life, as needed for cost-effectiveness studies. This utility measure is suitable for cost-effectiveness studies that are designed to 
Table 5 Multivariable Survey Regression Analysis to Estimate the SF-6D of Asthma Patients by Severity, 2010-2016

\begin{tabular}{|c|c|c|c|}
\hline & \multicolumn{3}{|l|}{ SF-6D } \\
\hline & Coefficient & SE & P-value \\
\hline \multicolumn{4}{|l|}{$\begin{array}{l}\text { Asthma severity (ref: mild } \\
\text { asthma) }\end{array}$} \\
\hline Moderate & -0.003 & 0.005 & 0.482 \\
\hline Severe & -0.059 & 0.017 & 0.001 \\
\hline ED visits & -0.039 & 0.005 & $<0.001$ \\
\hline Hospitalizations & -0.042 & 0.007 & $<0.001$ \\
\hline \multicolumn{4}{|l|}{ Age (years) (ref: 18-34) } \\
\hline $35-64$ & -0.054 & 0.005 & $<0.001$ \\
\hline$\geq 65$ & -0.023 & 0.008 & 0.003 \\
\hline \multicolumn{4}{|l|}{ Sex (ref: male) } \\
\hline Female & -0.020 & 0.005 & $<0.001$ \\
\hline \multicolumn{4}{|l|}{ Race (ref: White) } \\
\hline Black & 0.033 & 0.005 & $<0.001$ \\
\hline Other ${ }^{a}$ & -0.006 & 0.008 & 0.492 \\
\hline \multicolumn{4}{|l|}{ Hispanic (ref: no) } \\
\hline Yes & 0.018 & 0.006 & 0.001 \\
\hline \multicolumn{4}{|l|}{ Region (ref: northwest) } \\
\hline Midwest & -0.020 & 0.007 & 0.005 \\
\hline South & -0.022 & 0.007 & 0.001 \\
\hline West & -0.021 & 0.007 & 0.006 \\
\hline \multicolumn{4}{|l|}{ Marital status (ref: not married) } \\
\hline Married & -0.004 & 0.088 & 0.630 \\
\hline Widowed & -0.009 & 0.007 & 0.202 \\
\hline Divorced/separated & 0.010 & 0.006 & 0.107 \\
\hline \multicolumn{4}{|l|}{ Education (ref: advanced degree) } \\
\hline Less than high school diploma & -0.046 & 0.008 & $<0.001$ \\
\hline High school graduate & -0.025 & 0.008 & 0.001 \\
\hline Bachelor's degree & -0.021 & 0.007 & 0.002 \\
\hline \multicolumn{4}{|l|}{ Income level (ref: >80,000) } \\
\hline$\leq 20,000$ & -0.076 & 0.007 & $<0.001$ \\
\hline$>20,000-40,000$ & -0.050 & 0.006 & $<0.001$ \\
\hline$>40,000-80,000$ & -0.023 & 0.005 & $<0.001$ \\
\hline \multicolumn{4}{|l|}{ Insurance (ref: uninsured) } \\
\hline Any private & 0.037 & 0.009 & $<0.001$ \\
\hline Public only & -0.038 & 0.010 & $<0.001$ \\
\hline Smoking & -0.031 & 0.007 & $<0.001$ \\
\hline $\mathrm{CCl}^{\mathrm{b}}$ & -0.021 & 0.002 & $<0.001$ \\
\hline Intercept & 0.872 & 0.013 & $<0.001$ \\
\hline
\end{tabular}

Notes: ${ }^{a}$ Other includes American Indian, Alaska Native, Asian, Native Hawaiian, Pacific Islander, and multiple races. ${ }^{b}$ Charlson Comorbidity Index includes myocardial infarction, heart failure, peripheral vascular disease, dementia, cerebrovascular disease, connective tissue disease, ulcer disease mild liver disease hemiplegia, moderate or severe renal disease, diabetes, tumor, moderate or severe liver, and metastatic solid tumor.

Abbreviations, SF-6D, short-form six-dimension; PCS, physical component summary; MCS, mental component summary; SE, standard error; ED, emergency department; $\mathrm{CCl}$, charlson comorbidity index. capture the benefits of treatments that include different levels of asthma severity and various patient characteristics. Whereas, general HRQOL measures can be used to calculate health utilities and allow utility weights of different diseases to be compared. Importantly, the utility weight of patients with severe asthma was 0.659 in our analysis, which is similar to the utilities of patients with communityacquired pneumococcal pneumonia or type 2 diabetes experiencing heart failure $(0.677)$ or stroke $(0.621)$ which are known to induce substantial deterioration of patients' QOL and impose burden on caregivers. ${ }^{39,40}$

In agreement with previous studies using asthma-specific (ie, AQLQ) or generic HRQOL measures (ie, EQ-5D), ${ }^{5,26,41}$ our regression models suggested that in addition to severe asthma, older age, female, lower level education, lower household income, having public insurance, smoking, and higher CCI scores were significantly related with worse health utilities (ie, SF-6D). We also found that ED visits and hospitalizations were significantly associated with utility decrements after controlling for asthma severity and other demographic and clinical variables. ${ }^{35,42}$ When risk factors associated with poor HRQOL among patients with asthma were subdivided into physical and mental health, notably, our results of SF-12 PCS and MCS suggest that female, older age, and less education were associated with poor physical health, while mental health was not significantly associated with these factors. It may be that physical activity is reduced in adults with asthma, particularly in female and older aged asthma patients ${ }^{43}$ and the negative association between vigorous physical activity and wheezing is more often found in women. ${ }^{44}$ In addition, lower health literacy has been reported in patients with a lower education level. Lower health literacy can cause more delayed diagnosis of asthma, poorer access to health care, and less adherence to healthy lifestyles, which results in the association with worse physical function and asthma-related QOL. ${ }^{26,45}$

Our study has several limitations. The MEPS survey lacks physiological data (eg, lung function and clinical measures of asthma severity) to corroborate our severity categories. A potential limitation is that the differences in HRQOL between moderate and severe asthma could be underestimated. The GINA guidelines use five management steps to define asthma severity, categorizing step 3 as moderate and steps 4 and 5 as severe asthma. However, we classified steps 3 and 4 as moderate and step 5 as severe, because the MEPS data has limited information on ICS/LABA to permit distinguishing between steps 3 and 4 based on ICS/LABA dose. This 
classification of asthma severity has been used in previous research, ${ }^{17-19}$ and we have a clearly defined severe asthma based upon added on medications (ie, tiotropium, omalizumab, mepolizumab, low dose OCS). Second, the level of asthma symptom control was not captured in measuring the HRQOL of patients with asthma by severity, as asthma severity was defined based on the controller medication treatment of the GINA guideline, although it is possible that asthma control may affect HRQOL impairment. ${ }^{5}$ Our results estimate the HRQOL of patients with mild, moderate, and severe asthma including all levels of symptom control. Third, we adjusted for covariates known to be associated with HRQOL; however, we were dependent on survey data so that there may have been some unmeasured confounders (eg, environmental stimuli) that were not reported or unavailable. Although unmeasured confounding factors were not adjusted, we included key confounding factors such as sociodemographic information and clinical variables that were considered in previous studies. ${ }^{5,26}$ Lastly, we could not incorporate HRQOL changes by asthma symptoms over time. We used HRQOL from one point in time in the MEPS data based on the cross-sectional nature of the survey. Bearing this limitation in mind, our results appear to suggest that asthma severity decreased HRQOL independent of demographics and clinical characteristics.

\section{Conclusion}

This study suggests that patients with asthma perceived that their HRQOL was impaired by asthma and that severe asthma substantially worsens HRQOL compared to mild asthma, particularly on the physical component of the SF12 instrument, in the general US population. Factors of female, older age, and low education also had a deleterious effect on physical HRQOL. These results suggest that efforts of asthma management of physical health in addition to mental health should focus on severe asthma, female, older aged, and less educated asthma patients to improve asthma HRQOL.

\section{Ethics and Consent Statement}

Medical Expenditure Panel Survey (MEPS) data are available to the public online (https://meps.ahrq.gov/data stats/ download data files.jsp), and the study used fully deidentified data. Therefore, Institutional Review Board approval was not required and consent for publication was not applicable for this work.

\section{Author Contributions}

All authors contributed to data analysis or interpretation, drafting or revising the article, have agreed on the journal to which the article will be submitted, gave final approval of the version to be published, and agree to be accountable for all aspects of the work.

\section{Funding}

Research reported in this publication was supported by the National Heart, Lung, and Blood Institute of the National Institutes of Health under Award Number R01HL136945. The content is solely the responsibility of the authors and does not necessarily represent the official views of the National Institutes of Health.

\section{Disclosure}

Hyun Jin Song, Kathryn V Blake, Debbie L Wilson, and Haesuk Park declare that they have no competing interests. Almut $\mathrm{G}$ Winterstein has received research funding from Merck Sharp Dohme Corp and personal fees from Arbor Pharmaceutical, outside the submitted work.

\section{References}

1. Global Initiative for Asthma (GINA). Global strategy for asthma management and prevention. 2020. Available from: https://ginasthma. org/wp-content/uploads/2020/04/GINA-2020-full-report_-final-_wms. pdf. Accessed March 24, 2020.

2. Nurmagambetov T, Kuwahara R, Garbe P. The Economic Burden of Asthma in the United States, 2008-2013. Ann Am Thorac Soc. 2018;15(3):348-356. doi:10.1513/AnnalsATS.201703-259OC

3. Stanescu S, Kirby SE, Thomas M, Yardley L, Ainsworth B. A systematic review of psychological, physical health factors, and quality of life in adult asthma. NPJ Prim Care Respir Med. 2019;29 (1):37. doi:10.1038/s41533-019-0149-3

4. Vollmer WM, Markson LE, O'Connor E, et al. Association of asthma control with health care utilization and quality of life. Am J Respir Crit Care Med. 1999;160:1647-1652. doi:10.1164/ajrccm.160 .5 .9902098

5. Chen H, Gould MK, Blanc PD, et al. Asthma control, severity, and quality of life: quantifying the effect of uncontrolled disease. J Allergy Clin Immunol. 2007;120:396-402. doi:10.1016/j.jaci.2007.04.040

6. Siroux V, Boudier A, Anto JM, et al. Quality-of-life and asthma-severity in general population asthmatics: results of the ECRHS II study. Allergy. 2008;63:547-554. doi:10.1111/j.13989995.2008.01638.x

7. Sullivan PW, Ghushchyan V. Preference-based EQ-5D index scores for chronic conditions in the United States. Med Decis Making. 2006;26 (4):410-420. doi:10.1177/0272989X06290495

8. Sullivan PW, Lawrence WF, Ghushchyan V. A National cadalog of preference-based scores for chronic conditions in the United States. Med Care. 2005;43(7):736-749. doi:10.1097/01.mlr.0000172050.67085.4f

9. Barton GR, Sach TH, Doherty M, Avery AJ, Jenkinson C, Muir KR. An assessment of the Discriminative ability of the EQ-5D index, SF-6D, and EQ VAS, using sociodemographic factors and clinical conditions. Eur J Health Econ. 2008;9(3):237-249. doi:10.1007/ s10198-007-0068-z 
10. Dean BB, Calimlim BC, Sacco P, et al. Uncontrolled asthma: assessing quality of life and productivity of children and their caregivers using a cross-sectional internet-based survey. Health Qual Life Outcomes. 2010;8:1-10. doi:10.1186/1477-7525-8-96

11. Hossny E, Caraballo L, Casale T, El-Gamal Y, Rosenwasser L. Severe asthma and quality of life. World Allergy Organ J. 2017;10 (1):28. doi:10.1186/s40413-017-0159-y

12. Garratt AM, Hutchinson A, Russell I. Patient-assessed measures of health outcome in asthma: a comparison of four approaches. Respir Med. 2000;94(6):597-606. doi:10.1053/rmed.2000.0787

13. Ware J, Kosinski M, Keller SD. A 12-Item Short-Form Health Survey: construction of scales and preliminary tests of reliability and validity. Med Care. 1996;34(3):220-233. doi:10.1097/ 00005650-199603000-00003

14. Demoly P, Annunziata K, Gubba E, Adamek L. Repeated cross-sectional survey of patient-reported asthma control in Europe in the past 5 years. Eur Respir Rev. 2012;21(123):66-74. doi:10.1183/09059180.00008111

15. Agency for Healthcare Research and Quality (AHRQ). Medical Expenditure Panel Survey (MEPS). Available from: https://meps. ahrq.gov/mepsweb/. Accessed March 24, 2020.

16. Global Initiative for Asthma (GINA). Global strategy for asthma management and prevention 2010-2016. Available from: https:// ginasthma.org/archived-reports/. Accessed March 24, 2020.

17. Kang HR, Song HJ, Nam JH, et al. Risk factors of asthma exacerbation based on asthma severity: a nationwide population-based observational study in South Korea. BMJ Open. 2018;8(3):e020825. doi:10.1136/bmjopen-2017-020825

18. Lee YJ, Kwon SH, Hong SH, et al. Health care utilization and direct costs in mild, moderate, and severe adult asthma: a descriptive study using the 2014 South Korean Health Insurance database. Clin Ther. 2017;39(3):527-536. doi:10.1016/j.clinthera.2017.01.025

19. Song HJ, Blake KV, Wilson DL, Winterstein AG, Park H. Medical Costs and Productivity Loss Due to Mild, Moderate, and Severe Asthma in the United States. J Asthma Allergy. 2020;13:545-555. doi:10.2147/JAA.S272681

20. Garratt A, Ruta D, Abdalla M, Russell I. SF 36 health survey questionnaire: II. Responsiveness to changes in health status in four common clinical conditions. Qual Health Care. 1994;3(4):186-192. doi:10.1136/qshc.3.4.186

21. Brazier JE, Roberts J. The estimation of a preference-based measure of health from the SF-12. Med Care. 2004;42(9):851-859. doi:10.1097/01.mlr.0000135827.18610.0d

22. Huo T, Guo Y, Shenkman E, Muller K. Assessing the reliability of the short form 12 (SF-12) health survey in adults with mental health conditions: a report from the wellness incentive and navigation (WIN) study. Health Qual Life Outcomes. 2018;16(1):34. doi: 10.1186/s12955-018-0858-2

23. Ware JE, Kosinski M, Turner-Bowker DM, Gandek B. How to Score Version 2 of the SF-12 ${ }^{\circledR}$ Health Survey (With a Supplement Documenting Version 1). Lincoln, RI: QualityMetric Incorporated; 2002.

24. Hanmer J. Predicting an SF-6D preference-based score using MCS and PCS scores from the SF-12 or SF-36. Value Health. 2009;12 (6):958-966. doi:10.1111/j.1524-4733.2009.00535.x

25. Sullivan PW, Smith KL, Ghushchyan VH, Globe DR, Lin SL, Globe G. Asthma in USA: its impact on health-related quality of life. $J$ Asthma. 2013;50(8):891-899. doi:10.3109/02770903.2 013.813035

26. Gonzalez-Barcala FJ, de la Fuente-cid R, Tafalla M, Nuevo J, Caamaño-Isorna F. Factors associated with health-related quality of life in adults with asthma, A cross-sectional study. Multidiscip Respir Med. 2012;7(1):32. doi:10.1186/2049-6958-7-32

27. Mukherjee B, Ou HT, Wang F, Erickson SR. A new comorbidity index: the health-related quality of life comorbidity index. J Clin Epidemiol. 2011;64(3):309-319. doi:10.1016/j.jclinepi.2010.01.025
28. Quan H, Sundararajan V, Halfon P, et al. Coding algorithms for defining comorbidities in ICD-9-CM and ICD-10 administrative data. Med Care. 2005;43(11):1130-1139. doi:10.1097/01. mlr.0000182534.19832.83

29. Adams RJ, Wilson DH, Taylor AW, et al. Psychological factors and asthma quality of life: a population based study. Thorax. 2004;59 (11):930-935. doi:10.1136/thx.2003.010256

30. Lange P, Parner J, Vestbo J, Schnohr P, Jensen G. A 15-year follow-up study of ventilatory function in adults with asthma. $N$ Engl J Med. 1998;339:1194-1200. doi:10.1056/ NEJM199810223391703

31. Voll-Aanerud M, Eagan TML, Plana E, et al. Respiratory Symptoms in Adults Are Related to Impaired Quality of Life, Regardless of Asthma and COPD: results From the European Community Respiratory Health Survey. Health Qual Life Outcomes. 2010;8:107. doi:10.1186/1477-7525-8-107

32. McDonald VM, Hiles SA, Jones KA, Clark VL, Yorke J. Healthrelated quality of life burden in severe asthma. Med J Aust. 2018;209 (S2):S28-S33. doi:10.5694/mja18.00207

33. Taylor DR, Bateman ED, Boulet LP, et al. A new perspective on concepts of asthma severity and control. Eur Respir J. 2008;32 (3):545-554. doi:10.1183/09031936.00155307

34. Juniper EF, Wisniewski ME, Cox FM, Emmett AH, Nielsen KE, O'Byrne PM. Relationship between quality of life and clinical status in asthma: a factor analysis. Eur Respir J. 2004;23(2):287-291. doi:10.1183/09031936.04.00064204

35. Lloyd A, Price D, Brown R. The impact of asthma exacerbations on health-related quality of life in moderate to severe asthma patients in the UK. Prim Care Respir J. 2007;16(1):22-27. doi:10.3132/ pcrj.2007.00002

36. Kaambwa B, Chen G, Ratcliffe J, Iezzi A, Maxwell A, Richardson J. Mapping Between the Sydney Asthma Quality of Life Questionnaire (AQLQ-S) and Five Multi-Attribute Utility Instruments (MAUIs). Pharmacoeconomics. 2017;35(1):111-124. doi:10.1007/s40273-0160446-4

37. Apfelbacher CJ, Hankins M, Stenner P, Frew AJ, Smith HE. Measuring asthma-specific quality of life: structured review. Allergy. 2011;66(4):439-457. doi:10.1111/j.1398-9995.2010.02500.x

38. Juniper EF, Guyatt GH, Epstein RS, Ferrie PJ, Jaeschke R, Hiller TK. Evaluation of impairment of health related quality of life in asthma: development of a questionnaire for use in clinical trials. Thorax. 1992;47(2):76-83. doi:10.1136/thx.47.2.76

39. Andrade LF, Saba G, Ricard JD, et al. Health related quality of life in patients with community-acquired pneumococcal pneumonia in France. Health Qual Life Outcomes. 2018;16(1):28. doi:10.1186/ s12955-018-0854-6

40. Beaudet A, Clegg J, Thuresson PO, Lloyd A, McEwan P. Review of utility values for economic modeling in type 2 diabetes. Value Health. 2014;17(4):462-470. doi:10.1016/j.jval.2014.03.003

41. Hazell M, Frank T, Frank P. Health related quality of life in individuals with asthma related symptoms. Respir Med. 2003;97 (11):1211-1218. doi:10.1016/S0954-6111(03)00249-X

42. Uchmanowicz B, Panaszek B, Uchmanowicz I, Rosińczuk J. Clinical factors affecting quality of life of patients with asthma. Patient Prefer Adherence. 2016;10:579-589. doi:10.2147/PPA.S103043

43. Chen Y, Dales R, Krewski D. Leisure-time energy expenditure in asthmatics and non-asthmatics. Respir Med. 2001;95(1):13-18. doi:10.1053/rmed.2000.0961

44. Jerning C, Martinander E, Bjerg A, et al. Asthma and physical activity - a population based study results from the Swedish GA(2) LEN Survey. Respir Med. 2013;107(11):1651-1658. doi:10.1016/j. rmed.2013.08.040

45. Mancuso CA, Rincon M. Impact of health literacy on longitudinal asthma outcomes. J Gen Intern Med. 2006;21(8):813-817. doi:10.1111/j.1525-1497.2006.00528.x 


\section{Publish your work in this journal}

The Journal of Asthma and Allergy is an international, peer-reviewed open-access journal publishing original research, reports, editorials and commentaries on the following topics: Asthma; Pulmonary physiology; Asthma related clinical health; Clinical immunology and the immunological basis of disease; Pharmacological interventions and

Submit your manuscript here: https://www.dovepress.com/journal-of-asthma-and-allergy-journal new therapies. The manuscript management system is completely online and includes a very quick and fair peer-review system, which is all easy to use. Visit http://www.dovepress.com/testimonials.php to read real quotes from published authors. 